\title{
On a $q$-Difference Painlevé III Equation: I. Derivation, Symmetry and Riccati Type Solutions
}

\author{
Kenji KAJIWARA ${ }^{\dagger}$ and Kinji KIMURA $\ddagger$ \\ $\dagger$ Graduate School of Mathematics, Kyushu University, \\ 6-10-1 Hakozaki, Higashi-ku, Fukuoka 812-8512, Japan \\ E-mail:kaji@math.kyushu-u.ac.jp \\ $¥$ Department of Mathematics, Kobe University, 1-1 Rokko, Kobe 657-8501, Japan \\ E-mail: kimura@math.kobe-u.ac.jp
}

Received May 10, 2002; Revised July 25, 2002; Accepted July 31, 2002

\begin{abstract}
A $q$-difference analogue of the Painlevé III equation is considered. Its derivations, affine Weyl group symmetry, and two kinds of special function type solutions are discussed.
\end{abstract}

\section{Introduction}

Importance of the Painlevé equations is widely recognized in mathematics and mathematical physics, since the solutions plays a role of nonlinear version of special functions. So much studies for the Painlevé equations have been done from various points of view, such as classical solutions, asymptotics, geometric or algebraic structures and so on [13, 2]. On the other hand, discrete analogue of the Painlevé equations have been studied extensively after discovery of the singularity confinement property [4, which is a discrete analogue of the Painlevé property. What matters in studies of the discrete Painlevé equations was that they were in some sense too rich and looked almost uncontrollable. For example, number of known discrete Painlevé equations is several ten's, while that for the Painlevé equations is only six.

The situation has been changed after Sakai's theory [22]. Sakai constructed "space of initial conditions", or defining manifold of a discrete Painlevé equation called $q$-P $\mathrm{P}_{\mathrm{VI}}$ by blowing up $\mathbb{P}^{2}$ at nine points. Based on this fact, Sakai gave a classification of discrete Painlevé equations with the aid of theory of rational surfaces, and discussed the symmetries. The Painlevé equations appear naturally from the degeneration of defining manifold which is also interpreted as continuous limit. This theory gives us clear view to both discrete and continuous Painlevé equations. It also suggests the list of discrete Painlevé equations to be studied further. 
In this paper, we shall work with the following $q$-difference equation,

$$
\begin{aligned}
& \overline{f_{1}}=\frac{q^{2 N+1} c^{2}}{f_{0} f_{1}} \frac{1+a_{0} q^{n} f_{0}}{a_{0} q^{n}+f_{0}}, \\
& \underline{f_{0}}=\frac{q^{2 N+1} c^{2}}{f_{0} f_{1}} \frac{a_{1} q^{-n+\nu}+f_{1}}{1+a_{1} q^{-n+\nu} f_{1}},
\end{aligned}
$$

where $f_{i}=f_{i}(n ; \nu, N)(i=0,1)$ are dependent variables, $n \in \mathbb{Z}$ is the independent variable, $\nu, N \in \mathbb{Z}$ are parameters, and $q, a_{0}, a_{1}, c$ are constants. Moreover, $\overline{f_{i}}$ and $\underline{f_{i}}$ denote $f_{i}(n+1 ; \nu, N)$ and $f_{i}(n-1 ; \nu, N)$, respectively. We call equation (1.1) a $q$-difference Painlevé III equation $\left(q-\mathrm{P}_{\mathrm{III}}\right)$. Since $q-\mathrm{P}_{\mathrm{III}}$ appears in "Mul.6" in Sakai's classification, it is expected that $q-\mathrm{P}_{\text {III }}$ is a "good" equation.

The purpose of our work is to establish and to give explicit description for the following properties of $q$-P III (1.1):

- $q-\mathrm{P}_{\mathrm{III}}$ admits a continuous limit to the Painlevé III equation $\left(\mathrm{P}_{\mathrm{III}}\right)$,

$$
\frac{d^{2} v}{d x^{2}}=\frac{1}{v}\left(\frac{d v}{d x}\right)^{2}-\frac{1}{x} \frac{d v}{d x}+\frac{1}{x}\left(\alpha v^{2}+\beta\right)+\gamma v^{3}+\frac{\delta}{v}, \quad \gamma=-\delta=4,
$$

where $\alpha, \beta$ are parameters.

- $q$ - $\mathrm{P}_{\text {III }}$ is derived from the discrete-time Relativistic Toda lattice equation [23, while $\mathrm{P}_{\text {III }}$ is derived from the relativistic Toda lattice equation [21].

- $q$ - $\mathrm{P}_{\text {III }}$ can be regarded as a discrete dynamical system on the root lattice of type $A_{2} \times A_{1}$ [22, 9]. As a consequence, it admits symmetry of (extended) affine Weyl group of type $A_{1}^{(1)} \times A_{1}^{(1)}$ as the group of Bäcklund transformations.

- $q$-P $\mathrm{P}_{\text {III }}$ admits two classes of classical solutions, namely, the solutions which are reducible to discrete Riccati equation, and rational solutions. There are two kinds of Riccati type solutions, one of which is expressed by the Jackson's (modified) $q$-Bessel functions, while the Riccati type solution of $\mathrm{P}_{\mathrm{III}}$ is expressed by the (modified) Bessel functions [17.

- The rational solutions of $\mathrm{P}_{\text {III }}(1.2)$ are expressed as ratio of some special polynomials (Umemura polynomials). They admit determinant formula of Jacobi-Trudi type whose entries are given by the Laguerre polynomials [10]. The rational solutions of $q-\mathrm{P}_{\text {III }}$ are characterized by similar special polynomials which also admit determinant formula.

In this paper, being the first half of our work, we discuss the derivation, symmetry, and particular solutions of Riccati type. Rational solutions will be discussed in the next paper.

This paper is organized as follows. In Section 2 we derive $\mathrm{P}_{\mathrm{III}}$ and $q-\mathrm{P}_{\mathrm{III}}$ from the relativistic Toda lattice and discrete-time relativistic Toda lattice, respectively. Moreover, we also derive $q-\mathrm{P}_{\mathrm{III}}$ from the birational representation of affine Weyl group of type $A_{2}^{(1)} \times A_{1}^{(1)}$ which was presented in 9. In Section 3, we construct two kinds of particular solutions of Riccati type, one of which is expressed by Jackson's modified $q$-Bessel functions. We also construct determinant formula for these solutions. 
Remark 1. 1. Originally $q$-P $\mathrm{P}_{\mathrm{III}}$ (1.1) was derived in 12 as "asymmetric generalization" of $q$-difference Painlevé II equation $\left(q-\mathrm{P}_{\mathrm{II}}\right)$ [18,

$$
x_{n+1} x_{n} x_{n-1}=\xi \frac{x_{n}+\zeta \lambda^{n}}{1+\zeta \lambda^{n} x_{n}},
$$

where $\zeta, \xi$ are parameters, and $\lambda$ is a constant. In fact, putting

$$
\begin{aligned}
& q=\lambda^{-2}, \quad \nu=0, \quad a_{1}=\frac{1}{a_{0} \lambda}, \\
& x_{2 n}=f_{1}(n ; 0, N), \quad x_{2 n+1}=f_{0}(n ; 0, N),
\end{aligned}
$$

$q-\mathrm{P}_{\mathrm{III}}$ (1.1) is reduced to $q-\mathrm{P}_{\mathrm{II}}$ (1.3) with

$$
\xi=\lambda^{-4 N+2} c^{2}, \quad \zeta=\frac{1}{a_{0} \lambda} .
$$

2. The following $q$-difference equation,

$$
w_{n+1} w_{n-1}=\frac{c d\left(w_{n}-a q^{n}\right)\left(w_{n}-b q^{n}\right)}{\left(w_{n}-c\right)\left(w_{n}-d\right)},
$$

is also identified as a $q$-difference Painlevé III equation [18, 19]. Here, $q$ is a constant and $a, b, c, d$ are parameters. A class of particular solutions in terms of Jackson's $q$-Bessel functions is constructed in [11]. It looks that equation (1.6) has a different nature compared to our $q$-P $\mathrm{PIII}_{\text {(1.1) }}$, and it may be natural to regard equation (1.6) as a degenerate case of the $q$-difference Painlevé VI equation $\left(q-\mathrm{P}_{\mathrm{VI}}\right)$ [7, 22],

$$
\begin{aligned}
& y_{n} y_{n+1}=\frac{a_{3} a_{4}\left(z_{n+1}-b_{1} q^{n}\right)\left(z_{n+1}-b_{2} q^{n}\right)}{\left(z_{n+1}-b_{3}\right)\left(z_{n+1}-b_{4}\right)}, \\
& z_{n} z_{n+1}=\frac{b_{3} b_{4}\left(y_{n}-a_{1} q^{n}\right)\left(y_{n}-a_{2} q^{n}\right)}{\left(y_{n}-a_{3}\right)\left(y_{n}-a_{4}\right)}, \quad \frac{b_{1} b_{2}}{b_{3} b_{4}}=q \frac{a_{1} a_{2}}{a_{3} a_{4}},
\end{aligned}
$$

where $a_{i}$ and $b_{i}(i=1,2,3,4)$ are parameters.

\section{Derivations of $q-\mathrm{P}_{\mathrm{III}}$}

\subsection{Derivation of $\mathrm{P}_{\mathrm{III}}$ from relativistic Toda lattice}

In this section we derive $\mathrm{P}_{\mathrm{III}}(1.2)$ from the relativistic Toda lattice equation [21, 23,

$$
\begin{aligned}
& \frac{d}{d t} d_{n}=d_{n}\left(c_{n}-c_{n-1}\right), \\
& \frac{d}{d t} c_{n}=c_{n}\left(d_{n+1}+c_{n+1}-d_{n}-c_{n-1}\right), \quad n \in \mathbb{Z} .
\end{aligned}
$$

We introduce the variables $V_{n}$ and $K_{n}$ by

$$
c_{n}=-\frac{V_{n} K_{n}}{K_{n-1}}, \quad d_{n}=-K_{n-1}+\frac{V_{n-1} K_{n-1}}{K_{n-2}},
$$


which yields

$$
\begin{aligned}
& \frac{d}{d t} \log V_{n}=\left(K_{n-1}+V_{n-1}\right)-\left(K_{n}+V_{n}\right), \\
& \frac{d}{d t} K_{n}=K_{n} V_{n}-K_{n+1} V_{n+1}, \quad n \in \mathbb{Z} .
\end{aligned}
$$

We next impose two-periodicity on equation (2.3). Then we have,

$$
\begin{aligned}
& \frac{d}{d t} \log V_{0}=\left(K_{1}+V_{1}\right)-\left(K_{0}+V_{0}\right), \\
& \frac{d}{d t} \log V_{1}=\left(K_{0}+V_{0}\right)-\left(K_{1}+V_{1}\right), \\
& \frac{d}{d t} K_{0}=K_{0} V_{0}-K_{1} V_{1}, \\
& \frac{d}{d t} K_{1}=K_{1} V_{1}-K_{0} V_{0} .
\end{aligned}
$$

In order to derive $\mathrm{P}_{\mathrm{III}}(\mathbf{1 . 2})$, we introduce additional constants $\alpha_{n}(n=0,1)$ in such a way that

$$
\begin{aligned}
& \frac{d}{d t} \log V_{0}=\left(K_{1}+V_{1}\right)-\left(K_{0}+V_{0}\right)+\alpha_{0}, \\
& \frac{d}{d t} \log V_{1}=\left(K_{0}+V_{0}\right)-\left(K_{1}+V_{1}\right)+\alpha_{1}, \\
& \frac{d}{d t} K_{0}=K_{0} V_{0}-K_{1} V_{1}, \\
& \frac{d}{d t} K_{1}=K_{1} V_{1}-K_{0} V_{0} .
\end{aligned}
$$

We note that integrability is still kept under this generalization in a sense that it admits the Lax pair. It is easy to see from equation (2.5) that we have $K_{0}+K_{1}=$ const and $\frac{d}{d t} \log \left(V_{0} V_{1}\right)=\alpha_{0}+\alpha_{1}$. We normalize them so that

$$
\alpha_{0}+\alpha_{1}=1, \quad V_{0} V_{1}=\mathrm{e}^{t}, \quad K_{0}+K_{1}=\beta_{0} .
$$

Under this normalization, one can write down the equations for $V_{0}$ and $K_{1}$. Eliminating $K_{1}$, we obtain

$$
\frac{d^{2} V_{0}}{d t^{2}}=\frac{1}{V_{0}}\left(\frac{d V_{0}}{d t}\right)^{2}+V_{0}^{3}-\left(\alpha_{0}+\beta_{0}\right) V_{0}^{2}+\left(\beta_{0}-\alpha_{0}+1\right) \mathrm{e}^{t}-\frac{\mathrm{e}^{2 t}}{V_{0}}
$$

or

$$
\frac{d^{2} V_{0}}{d s^{2}}=\frac{1}{V_{0}}\left(\frac{d V_{0}}{d s}\right)^{2}-\frac{1}{s} \frac{d V_{0}}{d s}+\frac{V_{0}^{2}}{s^{2}}\left(V_{0}-\left(\alpha_{0}+\beta_{0}\right)\right)+\frac{\beta_{0}-\alpha_{0}+1}{s}-\frac{1}{V_{0}},
$$

where $s=\mathrm{e}^{t}$, which is a version of $\mathrm{P}_{\mathrm{III}}$ (named as " $\mathrm{P}_{\mathrm{III}}$ " in [17]). Equation (2.8) is rewritten into the ordinary form of $\mathrm{P}_{\mathrm{III}}$ (1.2) with $\alpha=-4\left(\alpha_{0}+\beta_{0}\right), \beta=4\left(-\alpha_{0}+\beta_{0}+1\right)$, $\gamma=-\delta=4$ by putting $s=x^{2}$ and $V_{0}=x v$. 
Remark 2. Without introducing additional constants $\alpha_{n}$, we obtain the equation for $V_{0}$ as

$$
\frac{d^{2} V_{0}}{d t^{2}}=\frac{1}{V_{0}}\left(\frac{d V_{0}}{d t}\right)^{2}+V_{0}^{3}-\beta_{0} V_{0}^{2}+\beta_{0}-\frac{1}{V_{0}} .
$$

Equation (2.9) is classified as "XII" in Gambier's classification [6], and integrated in terms of the elliptic functions. Therefore, such generalization of the relativistic Toda lattice equation is crucial in order to derive $\mathrm{P}_{\mathrm{III}}$. Such situation can be seen for other Painlevé equations, where they are derived by the similar generalization of proper soliton equations 1, 15, 16, 14.

\subsection{Derivation of $q-\mathrm{P}_{\mathrm{III}}$ from discrete-time relativistic Toda lattice}

Let us next consider the discrete case. We start from the discrete-time relativistic Toda lattice equation [23] in the following form,

$$
\begin{array}{ll}
\frac{\bar{d}_{n}}{d_{n}}=\frac{a_{n-1}-d_{n-1}}{a_{n}-d_{n}}, & \frac{\bar{c}_{n}}{c_{n}}=\frac{a_{n-1}+c_{n-1}}{a_{n}+c_{n}}, \\
a_{n}=1+d_{n}+\frac{c_{n+1}}{a_{n+1}}, & n \in \mathbb{Z},
\end{array}
$$

where $a_{n}=a_{n}(t), c_{n}=c_{n}(t), d_{n}=d_{n}(t)$ and ${ }^{-}$denotes the value at $t+1$. We note that for convenience we have inverted the space direction $n$ of the original equation in [23. We introduce the variables $K_{n}=K_{n}(t), V_{n}=V_{n}(t)$ by

$$
\frac{c_{n}}{a_{n}}=\bar{V}_{n}, \quad a_{n}=K_{n}
$$

Then equation (2.10) is rewritten as

$$
\begin{aligned}
& \frac{\bar{V}_{n}}{V_{n}}=\frac{K_{n-1}+V_{n-1}}{K_{n}+V_{n}}, \\
& \frac{\bar{K}_{n+1}}{K_{n}}=\frac{1+\bar{V}_{n}}{1+\bar{V}_{n+1}} \frac{\bar{K}_{n+1}+\bar{V}_{n+1}}{\bar{K}_{n}+\bar{V}_{n}} .
\end{aligned}
$$

We next impose two-periodicity on equation (2.12), which yields

$$
\begin{aligned}
& \frac{\bar{V}_{0}}{V_{0}}=\frac{K_{1}+V_{1}}{K_{0}+V_{0}}, \quad \frac{\bar{V}_{1}}{V_{1}}=\frac{K_{0}+V_{0}}{K_{1}+V_{1}}, \\
& \frac{\bar{K}_{1}}{K_{0}}=\frac{1+\bar{V}_{0}}{1+\bar{V}_{1}} \frac{\bar{K}_{1}+\bar{V}_{1}}{\bar{K}_{0}+\bar{V}_{0}}, \quad \frac{\bar{K}_{0}}{K_{1}}=\frac{1+\bar{V}_{1}}{1+\bar{V}_{0}} \frac{\bar{K}_{0}+\bar{V}_{0}}{\bar{K}_{1}+\bar{V}_{1}} .
\end{aligned}
$$

Similarly to the continuous case, we introduce additional constants $a_{n}(n=0,1)$ in such a way that

$$
\begin{aligned}
& \frac{\bar{V}_{0}}{V_{0}}=\frac{K_{1}+V_{1}}{K_{0}+V_{0}} a_{0}, \quad \frac{\bar{V}_{1}}{V_{1}}=\frac{K_{0}+V_{0}}{K_{1}+V_{1}} a_{1}, \\
& \frac{\bar{K}_{1}}{K_{0}}=\frac{1+\bar{V}_{0}}{1+\bar{V}_{1}} \frac{\bar{K}_{1}+\bar{V}_{1}}{\bar{K}_{0}+\bar{V}_{0}}, \quad \frac{\bar{K}_{0}}{K_{1}}=\frac{1+\bar{V}_{1}}{1+\bar{V}_{0}} \frac{\bar{K}_{0}+\bar{V}_{0}}{\bar{K}_{1}+\bar{V}_{1}} .
\end{aligned}
$$


We remark equation (2.14) is still integrable in a sense that it passes the singularity confinement test [4. It is easy to see from equation (2.14) that we have $\bar{K}_{0} \bar{K}_{1}=K_{0} K_{1}=$ const and $\bar{V}_{0} \bar{V}_{1}=a_{0} a_{1} V_{0} V_{1}$. We normalize as

$$
a_{0} a_{1}=q^{2}, \quad V_{0} V_{1}=c_{0}^{2} q^{2 t}, \quad K_{0} K_{1}=b_{0}^{2},
$$

where $b_{0}$ and $c_{0}$ are constants. Under this normalization, we obtain the equation,

$$
\bar{X} Y X=\frac{a_{0}}{q} \frac{1+c_{0} q q^{t} Y}{Y+c_{0} q q^{t}}, \quad Y X \underline{Y}=\frac{a_{0}}{q} \frac{X+\frac{b_{0}}{c_{0}} q^{-t}}{1+\frac{b_{0}}{c_{0}} q^{-t} X},
$$

where the variables $X=X(t)$ and $Y=Y(t)$ are defined by

$$
X=-\frac{a_{0} c_{0}}{b_{0}} q^{t} \frac{K_{1}}{\bar{V}_{0}}, \quad Y=\frac{1}{c_{0} q} q^{-t} \bar{V}_{0},
$$

respectively, and _ denotes the value at $t-1$. Equation (2.16) is essentially the same as $q-\mathrm{P}_{\text {III (1.1). }}$.

We finally comment on the continuous limit of $q-\mathrm{P}_{\text {III }}$ to $\mathrm{P}_{\mathrm{III}}$. The simplest way is to take the limit on the level of equation (2.14); replacing $t, K_{i}, V_{i}$ and $\alpha_{i}$ by $t / h, 1+h K_{i}, h V_{i}$ and $1+\alpha_{i} h(i=0,1)$, respectively, and taking the limit $h \rightarrow 0$, we obtain equation (2.5).

\subsection{Birational representation of affine Weyl group $\widetilde{W}\left(A_{1}^{(1)} \times A_{2}^{(1)}\right)$}

In [9], the following birational representation of (extended) affine Weyl group $\widetilde{W}\left(A_{1}^{(1)} \times\right.$ $\left.A_{2}^{(1)}\right)$ is presented:

Theorem $1([\mathbf{9}])$. Let $\boldsymbol{C}\left(a_{i}, f_{i}(i=0,1,2)\right)$ be the field of rational functions in $a_{i}$ and $f_{i}$. We define the transformations $s_{i}(i=0,1,2)$ and $\pi$ acting on $\boldsymbol{C}\left(a_{i}, f_{i}(i=0,1,2)\right)$ by

$$
\begin{aligned}
& s_{i}\left(a_{j}\right)=a_{j} a_{i}^{-a_{i j}}, \quad s_{i}\left(f_{j}\right)=f_{j}\left(\frac{a_{i}+f_{i}}{1+a_{i} f_{i}}\right)^{u_{i j}} \quad(i, j=0,1,2), \\
& \pi\left(x_{i}\right)=x_{i+1}, \quad(x=a, f), \quad i \in \mathbb{Z} / 3 \mathbb{Z},
\end{aligned}
$$

respectively, where $A=\left(a_{i j}\right)_{i, j=0}^{2}$ is the generalized Cartan matrix of type $A_{2}^{(1)}$ and $U=$ $\left(u_{i j}\right)_{i, j=0}^{2}$ is an orientation matrix of the corresponding Dynkin diagram:

$$
A=\left[\begin{array}{ccc}
2 & -1 & -1 \\
-1 & 2 & -1 \\
-1 & -1 & 2
\end{array}\right], \quad U=\left[\begin{array}{ccc}
0 & 1 & -1 \\
-1 & 0 & 1 \\
1 & -1 & 0
\end{array}\right]
$$

Moreover, we define the transformations $w_{0}, w_{1}$ and $r$ by

$$
\begin{aligned}
& w_{0}\left(f_{i}\right)=\frac{a_{i} a_{i+1}\left(a_{i-1} a_{i}+a_{i-1} f_{i}+f_{i-1} f_{i}\right)}{f_{i-1}\left(a_{i} a_{i+1}+a_{i} f_{i+1}+f_{i} f_{i+1}\right)}, \\
& w_{1}\left(f_{i}\right)=\frac{1+a_{i} f_{i}+a_{i} a_{i+1} f_{i} f_{i+1}}{a_{i} a_{i+1} f_{i+1}\left(1+a_{i-1} f_{i-1}+a_{i-1} a_{i} f_{i-1} f_{i}\right)}, \\
& r\left(f_{i}\right)=\frac{1}{f_{i}}, \quad w_{0}\left(a_{i}\right)=w_{1}\left(a_{i}\right)=r\left(a_{i}\right)=a_{i}, \quad(i \in \mathbb{Z} / 3 \mathbb{Z}) .
\end{aligned}
$$


K Kajiwara and K Kimura

Then, the transformations $\left\langle s_{0}, s_{1}, s_{2}, \pi, w_{0}, w_{1}, r\right\rangle$ generate the extended affine Weyl group of type $A_{1}^{(1)} \times A_{2}^{(1)}$. Namely, these transformations satisfy the following fundamental relations,

$$
\begin{aligned}
& s_{i}^{2}=1, \quad\left(s_{i} s_{i+1}\right)^{3}=1, \quad \pi^{3}=1, \quad \pi s_{i}=s_{i+1} \pi \quad(i=0,1,2), \\
& w_{0}^{2}=w_{1}^{2}=r^{2}=1, \quad r w_{0}=w_{1} r,
\end{aligned}
$$

and the actions of $\left\langle s_{0}, s_{1}, s_{2}, \pi\right\rangle$ and $\left\langle w_{0}, w_{1}, r\right\rangle$ commute with each other.

One can construct translations $T_{i}(i=1,2,3,4)$ by

$$
T_{1}=\pi s_{2} s_{1}, \quad T_{2}=s_{1} \pi s_{2}, \quad T_{3}=s_{2} s_{1} \pi, \quad T_{4}=r w_{0} .
$$

We note that these translations satisfy $T_{i} T_{j}=T_{j} T_{i}(i, j=1,2,3,4)$ and $T_{1} T_{2} T_{3}=1$. The actions of $T_{i}$ on $a_{i}$ and $c$ are given by

$$
\begin{array}{llll}
T_{1}\left(a_{0}\right)=q a_{0}, & T_{1}\left(a_{1}\right)=q^{-1} a_{1}, & T_{1}\left(a_{2}\right)=a_{2}, & T_{1}(c)=c, \\
T_{2}\left(a_{0}\right)=a_{0}, & T_{2}\left(a_{1}\right)=q a_{1}, & T_{2}\left(a_{2}\right)=q^{-1} a_{2}, & T_{2}(c)=c, \\
T_{4}\left(a_{0}\right)=a_{0}, & T_{4}\left(a_{1}\right)=a_{1}, & T_{4}\left(a_{2}\right)=a_{2}, & T_{4}(c)=q c,
\end{array}
$$

respectively. We also remark that $a_{0} a_{1} a_{2}=q$ and $f_{0} f_{1} f_{2}=q c^{2}$ are invariant with respect to the actions of $\widetilde{W}\left(A_{1}^{(1)} \times A_{2}^{(1)}\right)$ and $\widetilde{W}\left(A_{2}^{(1)}\right)$, respectively.

The above affine Weyl group actions determine three birational, commutative, discrete flows on root lattice of type $A_{1} \times A_{2}$. Regarding one of the directions as the "time evolution", other directions can be considered as those of Bäcklund (Schlesinger) transformations.

In [9], $T_{4}$ is chosen as the time evolution, whose explicit action is given as

$$
\begin{aligned}
T_{4}\left(f_{0}\right) & =a_{0} a_{1} f_{1} \frac{1+a_{2} f_{2}+a_{2} a_{0} f_{2} f_{0}}{1+a_{0} f_{0}+a_{0} a_{1} f_{0} f_{1}}, \quad T_{4}\left(f_{1}\right)=a_{1} a_{2} f_{2} \frac{1+a_{0} f_{0}+a_{0} a_{1} f_{0} f_{1}}{1+a_{1} f_{1}+a_{1} a_{2} f_{1} f_{2}}, \\
T_{4}\left(f_{2}\right) & =a_{2} a_{0} f_{0} \frac{1+a_{1} f_{1}+a_{1} a_{2} f_{1} f_{2}}{1+a_{2} f_{2}+a_{2} a_{0} f_{2} f_{0}} .
\end{aligned}
$$

Choosing one of the $A_{2}$ direction, for example $T_{1}$, as the time evolution, we have

$$
\begin{aligned}
& T_{1}\left(f_{1}\right)=f_{2} \frac{1+a_{0} f_{0}}{a_{0}+f_{0}}=\frac{q c^{2}}{f_{0} f_{1}} \frac{1+a_{0} f_{0}}{a_{0}+f_{0}}, \\
& T_{1}^{-1}\left(f_{0}\right)=f_{2} \frac{a_{1}+f_{1}}{1+a_{1} f_{1}}=\frac{q c^{2}}{f_{0} f_{1}} \frac{a_{1}+f_{1}}{1+a_{1} f_{1}} .
\end{aligned}
$$

Operating $T_{1}^{n} T_{2}^{\nu} T_{4}^{N}(n, \nu, N \in \mathbb{Z})$ on equations (2.25) and (2.26), $\operatorname{denoting} T_{1}^{n} T_{2}^{\nu} T_{4}^{N}\left(f_{i}\right)=$ $f_{i}(n ; \nu, N)(i=0,1,2)$, we obtain $q-\mathrm{P}_{\mathrm{IV}}$,

$$
\begin{aligned}
& \tilde{f}_{0}=a_{0} a_{1} q^{\nu} f_{1} \frac{1+a_{2} q^{-\nu} f_{2}+a_{2} a_{0} q^{n-\nu} f_{2} f_{0}}{1+a_{0} q^{n} f_{0}+a_{0} a_{1} q^{\nu} f_{0} f_{1}}, \\
& \tilde{f}_{1}=a_{1} a_{2} q^{-n} f_{2} \frac{1+a_{0} q^{n} f_{0}+a_{0} a_{1} q^{\nu} f_{0} f_{1}}{1+a_{1} q^{-n+\nu} f_{1}+a_{1} a_{2} q^{-n} f_{1} f_{2}}, \\
& \tilde{f}_{2}=a_{2} a_{0} q^{n-\nu} f_{0} \frac{1+a_{1} q^{n} f_{1}+a_{1} a_{2} q^{-n} f_{1} f_{2}}{1+a_{2} q^{-\nu} f_{2}+a_{2} a_{0} q^{n-\nu} f_{2} f_{0}},
\end{aligned}
$$

and $q-\mathrm{P}_{\mathrm{III}}$ (1.1), respectively. In equation (2.27), $\tilde{f}_{i}$ denote $\tilde{f}_{i}=f_{i}(n ; \nu, N+1)(i=0,1,2)$. 
Remark 3. By construction, $q-\mathrm{P}_{\mathrm{III}}$ (1.1) describes one of the Bäcklund transformations of $q-\mathrm{P}_{\mathrm{IV}}$ (2.27), and vice versa. Moreover, under the specialization (1.4), $q-\mathrm{P}_{\mathrm{IV}}$ (2.27) coincides with the Bäcklund transformation of $q-\mathrm{P}_{\text {II }}$ (1.3) proposed by Joshi et al [8].

$q$ - $\mathrm{P}_{\text {III }}$ admits the symmetry described by the affine Weyl group of type $A_{1}^{(1)} \times A_{1}^{(1)}$. In fact, we can easily verify the following fact:

Proposition 1. We define $w_{0}^{\prime}, w_{1}^{\prime}$ by

$$
w_{0}^{\prime}=s_{0} s_{1} s_{0}, \quad w_{1}^{\prime}=s_{2} .
$$

Then, $w_{0}^{\prime}$ and $w_{1}^{\prime}$ satisfy the fundamental relations of $W\left(A_{1}^{(1)}\right)$,

$$
\left(w_{0}^{\prime}\right)^{2}=\left(w_{1}^{\prime}\right)^{2}=1 \text {. }
$$

Moreover, the actions of $\left\langle w_{0}^{\prime}, w_{1}^{\prime}\right\rangle$ commute with the actions of $T_{1}$ and $\left\langle w_{0}, w_{1}, r\right\rangle$.

\section{Particular solutions of Riccati type}

\subsection{Solutions in terms of Jackson's modified $q$-Bessel functions}

Many of Painlevé and discrete Painlevé equations are known to admit 1-parameter family of particular solutions expressible in terms of $(q-)$ classical special functions. Such solutions are obtained by finding the special cases where the Painlevé or discrete Painlevé equations are reduced to the (discrete) Riccati equations.

For example, $q-\mathrm{P}_{\mathrm{IV}}$ (2.27) admits the following Riccati type solutions:

Proposition 2 ([9]). $q-P_{\mathrm{IV}}$ 2.27) admits particular solutions given by

$$
f_{0}=a_{0} c^{2} q^{n+2 N+1} \frac{G_{n}(N)}{G_{n}(N+1)}, \quad f_{1}=-\frac{q}{a_{0} q^{n}} \frac{G_{n}(N+1)}{G_{n}(N)}, \quad f_{2}=-1
$$

for $\nu=0$, where $G_{n}(N)$ is a function satisfying the contiguity relations

$$
\begin{aligned}
& G_{n+1}(N+1)=G_{n}(N+1)+a_{0}^{2} c^{2} q^{2 n+2 N+1} G_{n}(N), \\
& G_{n+1}(N)=G_{n}(N+1)+c^{2} q^{2 N+1} G_{n}(N) .
\end{aligned}
$$

In particular, equations (3.2) admit polynomial solutions in $q^{N+1 / 2} c$ for $a_{0}=q$, and the polynomials $H_{n}(x)=\left(q^{N+1 / 2} c\right)^{-n} G_{n}(N), x=\left(q^{N+1 / 2} c+q^{-N-1 / 2} c^{-1}\right) / 2$ are identified as the continuous $q$-Hermite polynomials [3].

In the above solutions, the variables $N$ and $n$ play a role of independent variable and parameter, respectively. Since $q-\mathrm{P}_{\mathrm{III}}$ describes a Bäcklund transformation of $q-\mathrm{P}_{\mathrm{IV}}$, the above solutions of $q-\mathrm{P}_{\text {IV }}$ can be also regarded as those for $q-\mathrm{P}_{\mathrm{III}}$ only by exchanging the role of $N$ and $n$. For notational simplicity, we introduce a parameter $\mu$ and a function $I_{\mu}(n)$ by

$$
q^{N+1 / 2} c=q^{\mu}, \quad I_{\mu}(n)=\left(q^{N+1 / 2} c\right)^{-n} G_{n}(N) .
$$


Then equations (3.2) are rewritten as

$$
\begin{aligned}
& I_{\mu+1}(n+1)=q^{-\mu} I_{\mu+1}(n)+a_{0}^{2} q^{2 n+\mu} I_{\mu}(n), \\
& I_{\mu}(n+1)=q^{-\mu} I_{\mu+1}(n)+q^{\mu} I_{\mu}(n),
\end{aligned}
$$

respectively. We note that the three-term relation of $I_{\mu}(n)$ with respect to $n$,

$$
I_{\mu}(n+2)=\left(q^{\mu}+q^{-\mu}\right) I_{\mu}(n+1)-\left(1-a_{0}^{2} q^{2 n}\right) I_{\mu}(n),
$$

is nothing but Jackson's $q$-modified Bessel equation [3].

Proposition 3. Let $I_{\mu}(n)$ be a function satisfying equation (3.4). Then

$$
f_{0}=q^{2 \mu} a_{0} \frac{I_{\mu}(n)}{I_{\mu+1}(n)}, \quad f_{1}=-\frac{q}{a_{0}} \frac{I_{\mu+1}(n)}{I_{\mu}(n)},
$$

satisfy $q-P_{\text {III }}$ (1.1) with $\nu=0$ and $q^{N+1 / 2} c=q^{\mu}$.

We remark here that essentially the same solution is also constructed in [5, 20]. By successive application of the Bäcklund transformation $T_{2}$ on these solutions, we obtain "higher-order" solutions for $\nu \in \mathbb{Z}_{>0}$. Moreover, such solutions admit the following determinant formula.

Theorem 2. Let $I_{\mu}(n)$ be a function satisfying equation 3.4). For each $\nu \in \mathbb{Z}_{\geq 0}$, we define a $\nu \times \nu$ determinant $\varphi_{\nu}^{\mu}(n)$ by

$$
\varphi_{\nu}^{\mu}(n)=\operatorname{det}\left(I_{\mu}(n-i+j)\right)_{1 \leq i, j \leq \nu} \quad\left(\nu \in \mathbb{Z}_{>0}\right), \quad \varphi_{0}^{\mu}(n)=1 .
$$

Then,

$$
f_{0}=a_{0} q^{2 \mu} \frac{\varphi_{\nu}^{\mu+1}(n) \varphi_{\nu+1}^{\mu}(n)}{\varphi_{\nu+1}^{\mu+1}(n) \varphi_{\nu}^{\mu}(n)}, \quad f_{1}=-\frac{q}{a_{0}} \frac{\varphi_{\nu+1}^{\mu+1}(n) \varphi_{\nu}^{\mu}(n-1)}{\varphi_{\nu}^{\mu+1}(n-1) \varphi_{\nu+1}^{\mu}(n)},
$$

satisfy $q$ - $P_{\text {III }}$ (1.1) with $\nu \in \mathbb{Z}_{\geq 0}$ and $q^{N+1 / 2} c=q^{\mu}$.

Since Theorem 2 is obtained by the interpretation of the case of $q-\mathrm{P}_{\mathrm{IV}}$ [9], we omit the proof. We also note that it is possible to construct similar determinant formula for negative $\nu[9$.

\section{$3.2 \quad q-\mathrm{P}_{\text {III }}$ specific solutions}

In the previous section, we have discussed such solutions that $n, \nu$ and $N$ (or $\mu$ ) played a role of independent variable, determinant size and parameter of the solution, respectively. It is possible to construct another Riccati type solution in which the roles of $\nu$ and $N$ (or $\mu$ ) are exchanged.

We first look for the special values of $N$ and $c$ which admit decoupling of $q-\mathrm{P}_{\text {III }}$ (1.1) into discrete Riccati equation. In fact, it is easy to verify the following proposition: 
Proposition 4. $q$ - $P_{\mathrm{III}}$ (1.1) is decoupled into the discrete Riccati equation,

$$
\bar{f}_{1}=-\frac{q^{-\nu+1}}{a_{0} a_{1}} \frac{1+a_{0} q^{n} f_{0}}{f_{0}}, \quad f_{1}=-a_{0} a_{1} q^{\nu} \frac{1}{a_{0} q^{n}+f_{0}},
$$

when $N=0$ and $c=1$.

Eliminating $f_{1}$ from equation (3.9), we obtain a discrete Riccati equation for $f_{0}$,

$$
\bar{f}_{0}=\frac{-a_{0} q^{n+1}+\left(a_{0}^{2} a_{1}^{2} q^{2 \nu-1}-a_{0}^{2} q^{2 n+1}\right) f_{0}}{1+a_{0} q^{n} f_{0}} .
$$

Standard linearization of equation $(\underline{3.10})$ gives the following result:

Proposition 5. Let $H_{\nu}(n)$ be a function satisfying the following linear difference equation,

$$
H_{\nu}(n+1)=\left[1+a_{0}^{2} a_{1}^{2} q^{2 \nu}-a_{0}^{2} q^{2 n}\right] H_{\nu}(n)-a_{0}^{2} a_{1}^{2} q^{2 \nu} H_{\nu}(n-1) .
$$

Then,

$$
\begin{aligned}
& f_{0}=\frac{1}{a_{0} q^{n}} \frac{H_{\nu}(n+1)-H_{\nu}(n)}{H_{\nu}(n)} \\
& f_{1}=-a_{0}^{2} a_{1} q^{n+\nu} \frac{H_{\nu}(n)}{H_{\nu}(n+1)-\left(1-a_{0}^{2} q^{2 n}\right) H_{\nu}(n)},
\end{aligned}
$$

satisfy $q$ - $P_{\text {III }}$ (1.1) for $N=0$ and $c=1$.

One can investigate the evolution with respect to $\nu$ by substituting the above expression into the Bäcklund transformations of $q-\mathrm{P}_{\mathrm{III}}$ described in Section 2.2. After some manipulation we arrive at the following result:

Theorem 3. Let $H_{\nu}(n)$ be a function satisfying the following contiguity relations,

$$
\begin{aligned}
& H_{\nu}(n+1)-H_{\nu}(n)=-a_{0}^{2} q^{2 n} H_{\nu-1}(n), \\
& H_{\nu+1}(n)-H_{\nu}(n)=-a_{0}^{2} a_{1}^{2} q^{2 \nu} H_{\nu}(n-1) .
\end{aligned}
$$

Then,

$$
f_{0}=-a_{0} q^{n} \frac{H_{\nu-1}(n)}{H_{\nu}(n)}, \quad f_{1}=\frac{1}{a_{0}^{2} a_{1} q^{n+\nu-2}} \frac{H_{\nu}(n)}{H_{\nu-1}(n-1)},
$$

satisfy $q-P_{\text {III }}$ (1.1) with $N=0$ and $c=1$.

Remark 4. As is expected from the symmetry described in Section 2.2, the contiguity relations (3.13) and (3.14) for $H_{\nu}(n)$ are symmetric with respect to $n$ and $\nu$. In particular, the three-term relation with respect to $\nu$ is obtained as

$$
H_{\nu+1}(n)=\left[1+a_{0}^{2} q^{2 n}-a_{0}^{2} a_{1}^{2} q^{2 \nu}\right] H_{\nu}(n)-a_{0}^{2} q^{2 n} H_{\nu-1}(n) .
$$


We obtain higher-order solutions in the form of rational functions in $H_{\nu}(n)$ by applying the Bäcklund transformation $T_{4}$ to the above solution. Furthermore, these solutions admit the following determinant formula:

Theorem 4. For each $N \in \mathbb{Z}_{\geq 0}$, we define an $N \times N$ determinant $\phi_{\nu}^{N}(n)$ by

$$
\phi_{\nu}^{N}(n)=\operatorname{det}\left(H_{\nu+i-1}(n+j-1)\right)_{1 \leq i, j \leq N} \quad\left(N \in \mathbb{Z}_{>0}\right), \quad \phi_{\nu}^{0}(n)=1 .
$$

Then,

$$
\begin{aligned}
f_{0} & =-a_{0} q^{n+2 N} \frac{\phi_{\nu-1}^{N+1}(n) \phi_{\nu}^{N}(n)}{\phi_{\nu}^{N+1}(n) \phi_{\nu-1}^{N}(n)} \\
f_{1} & =\frac{1}{a_{0}^{2} a_{1} q^{n+\nu+2 N-2}} \frac{\phi_{\nu}^{N+1}(n) \phi_{\nu-1}^{N}(n-1)}{\phi_{\nu-1}^{N+1}(n-1) \phi_{\nu}^{N}(n)},
\end{aligned}
$$

satisfy $q$ - $P_{\text {III }}$ (1.1) with $c=1, N \in \mathbb{Z}_{\geq 0}$.

We next prove Theorem 4. It is a direct consequence of the following "multiplicative formula" with respect to $\phi$.

Proposition 6. The following formulas holds.

$$
\begin{aligned}
& 1+a_{0} q^{n} f_{0}=q^{-2 N} \frac{\phi_{\nu}^{N+1}(n+1) \phi_{\nu-1}^{N}(n-1)}{\phi_{\nu}^{N+1}(n) \phi_{\nu-1}^{N}(n)}, \\
& 1+\frac{f_{0}}{a_{0} q^{n}}=-a_{0}^{2} a_{1}^{2} q^{2(\nu+N-1)} \frac{\phi_{\nu-1}^{N+1}(n-1) \phi_{\nu}^{N}(n+1)}{\phi_{\nu}^{N+1}(n) \phi_{\nu-1}^{N}(n)}, \\
& 1+a_{1} q^{-n+\nu} f_{1}=\frac{1}{a_{0}^{2} q^{2(n-1)}} \frac{\phi_{\nu}^{N+1}(n-1) \phi_{\nu-1}^{N}(n)}{\phi_{\nu-1}^{N+1}(n-1) \phi_{\nu}^{N}(n)}, \\
& 1+\frac{f_{1}}{a_{1} q^{-n+\nu}}=\frac{1}{a_{0}^{2} a_{1}^{2} q^{2(\nu-1)}} \frac{\phi_{\nu-1}^{N+1}(n) \phi_{\nu}^{N}(n-1)}{\phi_{\nu-1}^{N+1}(n-1) \phi_{\nu}^{N}(n)}
\end{aligned}
$$

In fact, it is an easy task to derive $q-\mathrm{P}_{\text {III }}$ by using the above multiplicative formulas and equation (3.18). Furthermore, Proposition 6 follows from the following proposition.

Proposition 7. $\phi_{\nu}^{N}(n)$ satisfies the following bilinear difference equations.

$$
\begin{gathered}
\phi_{\nu}^{N+1}(n) \phi_{\nu-1}^{N}(n)-a_{0}^{2} q^{2(n+N)} \phi_{\nu-1}^{N+1}(n) \phi_{\nu}^{N}(n) \\
=q^{-2 N} \phi_{\nu}^{N+1}(n+1) \phi_{\nu-1}^{N}(n-1), \\
q^{2 N} \phi_{\nu-1}^{N+1}(n) \phi_{\nu}^{N}(n)-a_{0}^{2} a_{1}^{2} q^{2(\nu+N-1)} \phi_{\nu-1}^{N+1}(n-1) \phi_{\nu}^{N}(n+1) \\
=\phi_{\nu}^{N+1}(n) \phi_{\nu-1}^{N}(n), \\
a_{0}^{2} q^{2(n-1)} \phi_{\nu-1}^{N+1}(n-1) \phi_{\nu}^{N}(n)-q^{-2 N} \phi_{\nu}^{N+1}(n) \phi_{\nu-1}^{N}(n-1) \\
=\phi_{\nu}^{N+1}(n-1) \phi_{\nu-1}^{N}(n), \\
q^{2(\nu-1)} \phi_{\nu-1}^{N+1}(n-1) \phi_{\nu}^{N}(n)-q^{-2 N} \phi_{\nu}^{N+1}(n) \phi_{\nu-1}^{N}(n-1) \\
=\phi_{\nu-1}^{N+1}(n) \phi_{\nu}^{N}(n-1) .
\end{gathered}
$$

For example, equation (3.19) is derived from equation (3.23) by dividing both sides by $\phi_{\nu}^{N+1}(n) \phi_{\nu-1}^{N}(n)$. Therefore, the proof of Theorem 4 is now reduced to that of Proposition 7 We will give it in the next section. 


\subsection{Proof of Proposition 7}

Our basic idea for proving Proposition 7 is the same as that was done in 9 . Bilinear difference equations are derived from the Plücker relations, which are quadratic identities among determinants whose columns are shifted. Therefore, we first construct such "difference formulas" that relate "shifted determinants" and $\phi_{\nu}^{N}(n)$, by using the contiguity relations of $H_{\nu}(n)$. We then derive bilinear difference equations with the aid of difference formulas from proper Plücker relations. We take equation (3.24) as an example to show this procedure explicitly. For other equations, see Appendix A.

We introduce a notation,

$$
\begin{aligned}
\phi_{\nu}^{N}(n) & =\left|\begin{array}{cccc}
H_{\nu}(n) & H_{\nu+1}(n) & \cdots & H_{\nu+N-1}(n) \\
H_{\nu}(n+1) & H_{\nu+1}(n+1) & \cdots & H_{\nu+N-1}(n+1) \\
\vdots & \vdots & \ddots & \vdots \\
H_{\nu}(n+N-1) & H_{\nu+1}(n+N-1) & \cdots & H_{\nu+N-1}(n+N-1)
\end{array}\right| \\
& =\left|\mathbf{0}_{n}, \mathbf{1}_{n}, \cdots, \mathbf{N}-\mathbf{1}_{n}\right|,
\end{aligned}
$$

where $\boldsymbol{k}_{n}$ denotes a column vector,

$$
\boldsymbol{k}_{n}=\left(\begin{array}{c}
H_{\nu+k}(n) \\
H_{\nu+k}(n+1) \\
\vdots
\end{array}\right)
$$

We note that we abbreviate the suffix $n$ when unnecessary.

We next construct the difference formula.

\section{Lemma 1 (Difference formula I).}

$$
\begin{aligned}
& |\mathbf{0}, \mathbf{1}, \cdots, \boldsymbol{N}-\mathbf{1}|=\phi_{\nu}^{N}(n), \\
& \left|\mathbf{0}_{n+1}, \mathbf{0}, \cdots, \boldsymbol{N}-\mathbf{2}\right|=\left(-a_{0}^{2} a_{1}^{2}\right)^{-(N-2)} q^{-(2 \nu+N-2)(N-1)} \phi_{\nu}^{N}(n+1), \\
& \left|\mathbf{1}_{n+1}, \mathbf{0}, \cdots, \boldsymbol{N}-\mathbf{2}\right|=\left(-a_{0}^{2} a_{1}^{2}\right)^{-(N-2)} q^{-(2 \nu+N-2)(N-1)} \phi_{\nu}^{N}(n+1) .
\end{aligned}
$$

Proof. Equation (3.29) is nothing but the definition equation (3.27). Subtracting the $(k-1)$-th column from the $k$-th column of the left hand side of equation (3.29) and using the contiguity relation (3.14) for $k=N, N-1, \ldots, 2$, we have

$$
\phi_{\nu}^{N}(n)=\left|\begin{array}{ccc}
\cdots & H_{\nu+N-2}(n) & H_{\nu+N-1}(n)-H_{\nu+N-2}(n) \\
\cdots & H_{\nu+N-2}(n+1) & H_{\nu+N-1}(n+1)-H_{\nu+N-2}(n+1) \\
\vdots & \vdots & \vdots \\
\cdots & H_{\nu+N-2}(n+N-1) & H_{\nu+N-1}(n+N-1)-H_{\nu+N-2}(n+N-1)
\end{array}\right|
$$




$$
\begin{aligned}
& =\left|\begin{array}{cccc}
\cdots & H_{\nu+N-2}(n) & -a_{0}^{2} a_{1}^{2} q^{2(\nu+N-2)} H_{\nu+N-2}(n-1) \\
\cdots & H_{\nu+N-2}(n+1) & -a_{0}^{2} a_{1}^{2} q^{2(\nu+N-2)} H_{\nu+N-2}(n) \\
\vdots & \vdots & & \vdots \\
\cdots & H_{\nu+N-2}(n+N-1) & -a_{0}^{2} a_{1}^{2} q^{2(\nu+N-2)} H_{\nu+N-2}(n+N-2)
\end{array}\right| \\
= & -a_{0}^{2} a_{1}^{2} q^{2(\nu+N-2)} \\
& \times\left|\begin{array}{cccc}
H_{\nu}(n) & \ldots & H_{\nu+N-2}(n) & H_{\nu+N-2}(n-1) \\
H_{\nu}(n+1) & \cdots & H_{\nu+N-2}(n+1) & H_{\nu+N-2}(n) \\
\vdots & \vdots & \vdots & \vdots \\
H_{\nu}(n+N-1) & \cdots & H_{\nu+N-2}(n+N-1) & H_{\nu+N-2}(n+N-2)
\end{array}\right| \\
= & \cdots\left(\begin{array}{cccc}
\left.-a_{0}^{2} a_{1}^{2}\right)^{N-1} q^{(2 \nu+N-2)(N-1)} \\
H_{\nu}(n) & H_{\nu}(n-1) & \cdots & H_{\nu+N-2}(n-1) \\
H_{\nu}(n+1) & H_{\nu}(n) & \cdots & H_{\nu+N-2}(n) \\
\vdots & \vdots & \vdots & \vdots \\
H_{\nu}(n+N-1) & H_{\nu}(n+N-2) & \cdots & H_{\nu+N-2}(n+N-2)
\end{array} \mid, \quad(3.32\right.
\end{aligned}
$$

which is nothing but equation (3.30). Moreover, subtracting the second column multiplied by $a_{0}^{2} a_{1}^{2} q^{2 \nu}$ from the first column of the right hand side of equation (3.32) and using the contiguity relation (3.14), we obtain

$$
\begin{aligned}
\phi_{\nu}^{N}(n) & =\left(-a_{0}^{2} a_{1}^{2}\right)^{N-1} q^{(2 \nu+N-2)(N-1)} \\
& \times\left|\begin{array}{cccc}
H_{\nu+1}(n) & H_{\nu}(n-1) & \cdots & H_{\nu+N-2}(n-1) \\
H_{\nu+1}(n+1) & H_{\nu}(n) & \cdots & H_{\nu+N-2}(n) \\
\vdots & \vdots & \vdots & \vdots \\
H_{\nu+1}(n+N-1) & H_{\nu}(n+N-2) & \cdots & H_{\nu+N-2}(n+N-2)
\end{array}\right|,
\end{aligned}
$$

which is nothing but equation (3.31).

Now consider the Plücker relation,

$$
\begin{aligned}
0= & \left|\mathbf{1}_{n+1}, \mathbf{1}, \cdots, \boldsymbol{N}-\mathbf{1}, \mathbf{N}\right| \times\left|\mathbf{0}, \mathbf{1}, \cdots, \boldsymbol{N}-\mathbf{1}, \varphi_{2}\right| \\
& -|\mathbf{0}, \mathbf{1}, \cdots, \boldsymbol{N}-\mathbf{1}, \boldsymbol{N}| \times\left|\mathbf{1}_{n+1}, \mathbf{1}, \mathbf{1}, \cdots, \boldsymbol{N}-\mathbf{1}, \varphi_{2}\right| \\
& -\left|\mathbf{1}, \cdots, \boldsymbol{N}-\mathbf{1}, \mathbf{N}, \varphi_{2}\right| \times\left|\mathbf{1}_{n+1}, \mathbf{0}, \mathbf{1}, \cdots, \boldsymbol{N}-\mathbf{1}\right|
\end{aligned}
$$

where $\varphi_{2}$ is the column vector,

$$
\varphi_{2}=\left(\begin{array}{c}
1 \\
0 \\
\vdots \\
0
\end{array}\right)
$$

We obtain equation (3.24) by applying Lemma 1 to equation (3.34) after expanding with respect to the column $\varphi_{2}$. This completes the proof of bilinear equation (3.24). 


\section{A Difference formulas and Plücker relations}

In this appendix, we provide with data which are necessary for the proof of Proposition 7 We first give the difference formulas.

Lemma 2 (Difference formula II).

$$
\begin{aligned}
& \left|\mathbf{0}^{\prime}, \mathbf{1}^{\prime}, \cdots, \boldsymbol{N}-\mathbf{1}^{\prime}\right|=\phi_{\nu}^{N}(n), \\
& \left|\mathbf{0}_{\nu+1}^{\prime}, \mathbf{0}^{\prime}, \cdots, \boldsymbol{N}-\mathbf{2}^{\prime}\right|=\left(-a_{0}^{2}\right)^{-(N-2)} q^{-(2 n+N-2)(N-1)} \phi_{\nu+1}^{N}(n), \\
& \left|\mathbf{1}_{\nu+1}^{\prime}, \mathbf{0}^{\prime}, \cdots, \boldsymbol{N}-\mathbf{2}^{\prime}\right|=\left(-a_{0}^{2}\right)^{-(N-2)} q^{-(2 n+N-2)(N-1)} \phi_{\nu+1}^{N}(n),
\end{aligned}
$$

where

$$
\boldsymbol{k}_{\nu}^{\prime}=\left(\begin{array}{c}
H_{\nu}(n+k) \\
H_{\nu+1}(n+k) \\
\vdots
\end{array}\right) .
$$

We next rewrite $\phi_{\nu}^{N}(n)$ in terms of Toeplitz determinant with respect to $n$ by using the contiguity relations (3.13) and (3.14) as

$$
\begin{aligned}
& \phi_{\nu}^{N}(n)=\left(-a_{0}^{2} a_{1}^{2}\right)^{N(N-1) / 2} q^{N(N-1)(3 \nu+N-2) / 3} \\
& \times\left|\begin{array}{cccc}
H_{\nu}(n) & H_{\nu}(n+1) & \cdots & H_{\nu}(n+N-1) \\
H_{\nu}(n-1) & H_{\nu}(n) & \cdots & H_{\nu}(n+N-2) \\
\vdots & \vdots & \ddots & \vdots \\
H_{\nu}(n-N+1) & H_{\nu}(n-N+2) & \cdots & H_{\nu}(n)
\end{array}\right| \\
& \equiv\left(-a_{0}^{2} a_{1}^{2}\right)^{N(N-1) / 2} q^{N(N-1)(3 \nu+N-2) / 3} \psi_{\nu}^{N}(n) .
\end{aligned}
$$

Then we obtain the third difference formula:

\section{Lemma 3 (Difference formula III).}

$$
\begin{aligned}
& |\overline{\mathbf{0}}, \overline{\mathbf{1}}, \cdots, \overline{\mathbf{N}-\mathbf{1}}|=\psi_{\nu}^{N}(n), \\
& \left|\widetilde{\mathbf{0}}_{\nu+1}, \overline{\mathbf{0}}, \cdots, \overline{\mathbf{N}-\mathbf{2}}\right|=\left(-a_{0}^{2}\right)^{-(N-2)} q^{-2(n-1)(N-1)} \psi_{\nu+1}^{N}(n), \\
& \left|\widetilde{\overline{\mathbf{1}}}_{\nu+1}, \overline{\mathbf{0}}, \cdots, \overline{\mathbf{N}-\mathbf{2}}\right|=\left(-a_{0}^{2}\right)^{-(N-2)} q^{-2(n-1)(N-1)} \psi_{\nu+1}^{N}(n),
\end{aligned}
$$

where

$$
\overline{\boldsymbol{k}}_{\nu}=\left(\begin{array}{c}
H_{\nu}(n+k) \\
H_{\nu}(n+k-1) \\
H_{\nu}(n+k-2) \\
\vdots
\end{array}\right), \quad \widetilde{\boldsymbol{k}}_{\nu}=\left(\begin{array}{c}
H_{\nu}(n+k) \\
q^{-2} H_{\nu}(n+k-1) \\
q^{-4} H_{\nu}(n+k-2) \\
\vdots
\end{array}\right) .
$$

Lemmas 2 and 3 are proved by applying the same procedure as that for Lemma 1 on the determinants,

$$
\phi_{\nu}^{N}(n)=\left|\begin{array}{cccc}
H_{\nu}(n) & H_{\nu}(n+1) & \cdots & H_{\nu}(n+N-1) \\
H_{\nu+1}(n) & H_{\nu+1}(n+1) & \cdots & H_{\nu+1}(n+N-1) \\
\vdots & \vdots & \ddots & \vdots \\
H_{\nu+N-1}(n) & H_{\nu+N-1}(n+1) & \cdots & H_{\nu+N-1}(n+N-1)
\end{array}\right|
$$


and

$$
\psi_{\nu}^{N}(n)=\left|\begin{array}{cccc}
H_{\nu}(n) & H_{\nu}(n+1) & \cdots & H_{\nu}(n+N-1) \\
H_{\nu}(n-1) & H_{\nu}(n) & \cdots & H_{\nu}(n+N-2) \\
\vdots & \vdots & \ddots & \vdots \\
H_{\nu}(n-N+1) & H_{\nu}(n-N+2) & \cdots & H_{\nu}(n)
\end{array}\right|,
$$

respectively, by using the contiguity relation (3.13).

The bilinear equations (3.23) -(3.26) are derived by considering proper Plücker relations. We finally give the list the Plücker relations and difference formulas which are necessary for the derivations. This completes the proof of Proposition 17 and thus Theorem 4

Equation (3.23):

Plücker relation

$$
\begin{aligned}
0= & \left|\widetilde{\mathbf{1}}_{\nu+1}, \overline{\mathbf{1}}, \cdots, \overline{\mathbf{N}-\mathbf{1}}, \overline{\mathbf{N}}\right| \times\left|\overline{\mathbf{0}}, \overline{\mathbf{1}}, \cdots, \overline{\mathbf{N}-\mathbf{1}}, \varphi_{2}\right| \\
& -|\overline{\mathbf{0}}, \overline{\mathbf{1}}, \cdots, \overline{\mathbf{N}-\mathbf{1}}, \overline{\mathbf{N}}| \times\left|\widetilde{\overline{\mathbf{1}}}_{\nu+1}, \overline{\mathbf{1}}, \cdots, \overline{\mathbf{N}-\mathbf{1}}, \varphi_{2}\right| \\
& -\left|\overline{\mathbf{1}}, \cdots, \overline{\mathbf{N}-\mathbf{1}}, \overline{\mathbf{N}}, \varphi_{2}\right| \times\left|\widetilde{\overline{\mathbf{1}}}_{\nu+1}, \overline{\mathbf{0}}, \overline{\mathbf{1}}, \cdots, \overline{\mathbf{N}-\mathbf{1}}\right| .
\end{aligned}
$$

\section{Difference formula Lemma 3}

\section{Equation (3.25):}

Plücker relation

$$
\begin{aligned}
0= & \left|\mathbf{1}_{\nu+1}^{\prime}, \mathbf{1}^{\prime}, \cdots, N-\mathbf{1}^{\prime}, \mathbf{N}^{\prime}\right| \times\left|\mathbf{0}^{\prime}, \mathbf{1}^{\prime}, \cdots, N-\mathbf{1}^{\prime}, \varphi_{1}\right| \\
& -\left|\mathbf{0}^{\prime}, \mathbf{1}^{\prime}, \cdots, N-\mathbf{1}^{\prime}, \mathbf{N}^{\prime}\right| \times\left|\mathbf{1}_{\nu+1}^{\prime}, \mathbf{1}^{\prime}, \mathbf{1}^{\prime}, \cdots, \boldsymbol{N}-\mathbf{1}^{\prime}, \varphi_{1}\right| \\
& -\left|\mathbf{1}^{\prime}, \cdots, N-\mathbf{1}^{\prime}, \mathbf{N}^{\prime}, \varphi_{1}\right| \times\left|\mathbf{1}_{\nu+1}^{\prime}, \mathbf{0}^{\prime}, \mathbf{1}^{\prime}, \cdots, N-\mathbf{1}^{\prime}\right| .
\end{aligned}
$$

Difference formula Lemma 2

Equation (3.26):

Plücker relation

$$
\begin{aligned}
0= & \left|\mathbf{1}_{n+1}, \mathbf{1}, \cdots, \boldsymbol{N}-\mathbf{1}, \mathbf{N}\right| \times\left|\mathbf{0}, \mathbf{1}, \cdots, \mathbf{N}-\mathbf{1}, \varphi_{1}\right| \\
& -|\mathbf{0}, \mathbf{1}, \cdots, \boldsymbol{N}-\mathbf{1}, \mathbf{N}| \times\left|\mathbf{1}_{n+1}, \mathbf{1}, \mathbf{1}, \cdots, N-\mathbf{1}, \varphi_{1}\right| \\
& -\left|\mathbf{1}, \cdots, N-\mathbf{1}, \mathbf{N}, \varphi_{1}\right| \times\left|\mathbf{1}_{n+1}, \mathbf{0}, \mathbf{1}, \cdots, N-\mathbf{1}\right| .
\end{aligned}
$$

\section{Difference formula Lemma 1}

In the above data, $\varphi_{1}$ and $\varphi_{2}$ are column vectors given by

$$
\varphi_{1}=\left(\begin{array}{c}
0 \\
\vdots \\
0 \\
1
\end{array}\right), \quad \varphi_{2}=\left(\begin{array}{c}
1 \\
0 \\
\vdots \\
0
\end{array}\right)
$$




\section{References}

[1] Adler V E, Nonlinear Chains and Painlevé Equations, Physica D73 (1994), 335-351.

[2] Conte R (Editor), The Painlevé Property -One Century Later-, CRM Series in Mathematical Physics, Springer-Verlag, New York, 1999.

[3] Gasper G and Rahman M, Basic Hypergeometric Series, Encyclopedia of Mathematics and its Applications, Vol. 35, Cambridge University Press, Cambridge,1990.

[4] Grammaticos B, Ramani A and Papageorgiou V, Do Integrable Mappings Have the Painlevé Property?, Phys. Rev. Lett. 67 (1991), 1825-1828.

[5] Grammaticos B, Nijhoff F W, Papageorgiou V, Ramani A and Satsuma J, Linearization and Solutions of the Discrete Painlevé III Equation, Phys. Lett. A185 (1994), 446-452.

[6] Ince E L, Ordinary Differential Equations, Dover, New York, 1956.

[7] Jimbo M and Sakai H, A q-Analogue of the Sixth Painlevé Equation, Lett. Math. Phys. 38 (1996), 145-154.

[8] Joshi N, Ramani A and Grammaticos B, A Bilinear Approach to Discrete Miura Transformations, Phys. Lett. A249 (1998), 59-62.

[9] Kajiwara K, Noumi M and Yamada Y, A Study on the fourth q-Painlevé Equation, J. Phys. A: Math. Gen. 34 (2001), 8563-8581.

[10] Kajiwara K and Masuda T, On the Umemura Polynomials for the Painlevé III Equation, Phys. Lett. A260 (1999), 462-467.

[11] Kajiwara K, Ohta Y and Satsuma J, Casorati Determinant Solutions for the Discrete Painlevé III Equation, J. Math. Phys. 36 (1995), 4162-4174.

[12] Kruskal M D, Tamizhmani K M, Grammaticos B and Ramani A, Asymmetric Discrete Painlevé Equations, Regular and Chaotic Dynamics 5 (2000), 273-280.

[13] Levi D and Winternitz P (Editors), Painlevé Transcendents: Their Asymptotics and Physical Applications, NATO ASI series B278, Plenum, New York, 1992.

[14] Nijhoff F, Ramani A, Grammaticos B and Ohta Y, On Discrete Painlevé Equations Associated with the Lattice KdV Systems and the Painlevé VI Equation, Stud. Appl. Math. 106 (2000), 261-314.

[15] Noumi M and Yamada Y, Symmetries in the Fourth Painlevé Equation and Okamoto Polynomials, Nagoya Math. J. 153 (1999), 53-86.

[16] Noumi M and Yamada Y, Higher Order Painlevé Equations of Type $A_{l}^{(1)}$, Funkcial. Ekvac. 41 (1998), 483-503.

[17] Okamoto K, Studies on the Painlevé Equations. IV. Third Painlevé Equation $\mathrm{P}_{\mathrm{III}}$, Funkcial. Ekvac. 30 (1987), 305-332.

[18] Ramani A and Grammaticos B, Discrete Painlevé Equations: Coalescences, Limits and Degeneracies, Physica A228 (1996), 160-171.

[19] Ramani A, Grammaticos B and Hietarinta J, Discrete Versions of the Painlevé Equations, Phys. Rev. Lett. 67 (1991), 1829-1832. 
[20] Ramani A, Grammaticos B, Tamizhmani T and Tamizhmani K M, Special Function Solutions of the Discrete Painlevé Equations, Computers and Mathematics with Applications 42 (2001), 603-614.

[21] Ruijsenaars S N M, Relativistic Toda Systems, Commun. Math. Phys. 133 (1990), 217-247.

[22] Sakai H, Rational Surfaces Associated with Affine Root Systems and Geometry of the Painlevé Equations, Commun. Math. Phys. 220 (2001), 165-229.

[23] Suris Y, A Discrete-time Toda Lattice, J. Phys. A: Math. Gen. 29 (1996), 451-465. 